Capítulo 4

\title{
DiSEÑO DE UN PROGRAMA DE EJERCICIO PARA EL ADULTO MAYOR
}

María Victoria Quintero Cruz

Yisel Pinillos Patiño

A continuación se presenta el programa de ejercicios desarrollado en la investigación. El American College of Sports Medicine 2009 establece los parámetros en cuanto a la intensidad, frecuencia y duración de ejercicio para obtener beneficios para la salud (1).

1. La frecuencia semanal de ejercicio físico debe estar entre $3 \mathbf{y}$ 5 días a la semana.

2. La intensidad (según la fórmula de Karvonen) debe estar entre el 60 y el $90 \%$.

3. La duración del ejercicio debe ser entre 20 y 60 minutos.

La intensidad del ejercicio se basa en la fórmula Karvonen:

FC a un \% de intensidad = (FCmáx. - FC rep $)$ x (\% de I $)+$ FC rep

Donde:

- FCmáx (frecuencia cardiaca máxima) según Tanaka (2): 208 $(0,7 \mathrm{x}$ edad $)$

- FC rep (frecuencia cardiaca en reposo) 
- I es la intensidad de trabajo. Son los intervalos entre los que se tiene que mover el sujeto al realizar el ejercicio:

- Bajo nivel de trabajo cardiorrespiratorio: <60 \% FCmáx

- Nivel medio de trabajo cardiorrespiratorio: 60/70 \% FCmáx

- Buen nivel de trabajo cardiorrespiratorio: 70/80 \% FCmáx

Ejemplo:

La FC de entrenamiento para una persona con las siguientes características será:

Edad: 70 años

FC reposo: 75

$\%$ intensidad: $60 \%$

FC de entrenamiento $=($ FC máx. - FC rep $) \times(\%$ de I $)+$ FC rep

$\begin{array}{lllllll}=((208-(0.7 \times 70) & - & 75) & \times & (60 \%)+ & 75 \\ =((208-49) & - & 75) & X & (0,6)+ & 75 \\ =(159 & - & 75) & X & (0,6) & + & 75 \\ =84 & & & X & (0,6) & + & 75 \\ = & & & & + & 75\end{array}$

FC de entrenamiento $=125,4 \mathrm{pxm}$.

Según el cálculo anterior, esta persona no debe pasar de 125 pulsaciones por minuto durante la práctica del ejercicio.

Las dos primeras semanas se trabajaron con intensidades bajas (<50\%) como adaptación al ejercicio físico, las siguientes con 
intensidades medias (60\%), siempre teniendo en cuenta la frecuencia cardiaca de trabajo o de entrenamiento y la tolerancia al ejercicio del participante, el cual se monitoreó constantemente a través de la toma del pulso y la escala de esfuerzo de Borg (3).

Este programa de ejercicios se planeó para realizar 3 días a la semana y describe el trabajo a llevar a cabo en cada una de las sesiones día por día. Cada sesión de ejercicios consta de las siguientes partes:

\section{Fase inicial:}

1. Pre-monitoreo

2. Calentamiento aeróbico

\section{Fase central}

3. Calentamiento músculo-esquelético

4. Ejercicios de coordinación

5. Parte aeróbica

6. Ejercicios de fuerza

\section{Fase final:}

7. Enfriamiento o vuelta a la calma y relajación

8. Educación (puede hacerse al principio)

9. Recreación e integración social. 
Los ejercicios se basaron en los recomendados por Chávez Samperio (4), y en la experiencia de los investigadores. Se monitorizaron objetivamente mediante la toma del pulso al inicio, mitad y final del ejercicio, por parte del grupo de estudiantes y profesora encargados. Así mismo se usó la observación permanente del individuo de las respuestas físicas y mentales ante el estímulo del esfuerzo físico.

En cuanto al pulso se tuvo en cuenta que este no sobrepasara la frecuencia cardiaca de entrenamiento o frecuencia cardiaca de trabajo, además se mantuvo un ritmo e intensidad regulares. Durante el ejercicio el pulso se palpó durante 15 sgs. y se multiplicó por 4.

Para el presente programa además de la toma de pulso se tuvo como guía de monitoreo del esfuerzo del ejercicio la escala de Borg modificada (3), la cual se explicó hasta asegurarnos de que fue entendida por todos los participantes y se ubicó en un sitio visible.

El desarrollo del programa de ejercicio físico posibilitó una intervención de 36 sesiones de entrenamiento, distribuidas en cuatro microciclos. El método de entrenamiento fue el progresivo escalonado con cargas submáximas, entre el 55 y $65 \%$ en la fase general, entre el 65 y $75 \%$ en la fase específica. El medio utilizado fue 86 gravitacional, bajo una planificación de carácter tradicional, con el objetivo fundamentado en el componente de la carga, direccionado a desarrollar actividades de locomoción basadas en caminatas (FC de 75 a 85 \% CFI), fuerza y flexibilidad, como componentes específicos. Cada sesión se desarrolló según la dosis de ejercicio físico, la cual coincide con la que prescribe la guía específica diseñada para personas mayores sanas y que corresponde al American College Sports Medicine position stand (1). El tiempo de duración de las sesiones fue de 70 minutos, de los cuales 45 minutos se utilizaron 
en la fase central y el resto se distribuyó entre calentamiento y estiramiento. Al finalizar las 12 semanas se realizó la evaluación post-test para establecer los cambios en las variables de estudio.

Antes de iniciar el programa de ejercicios a cada participante se le calculó la frecuencia cardiaca de trabajo.

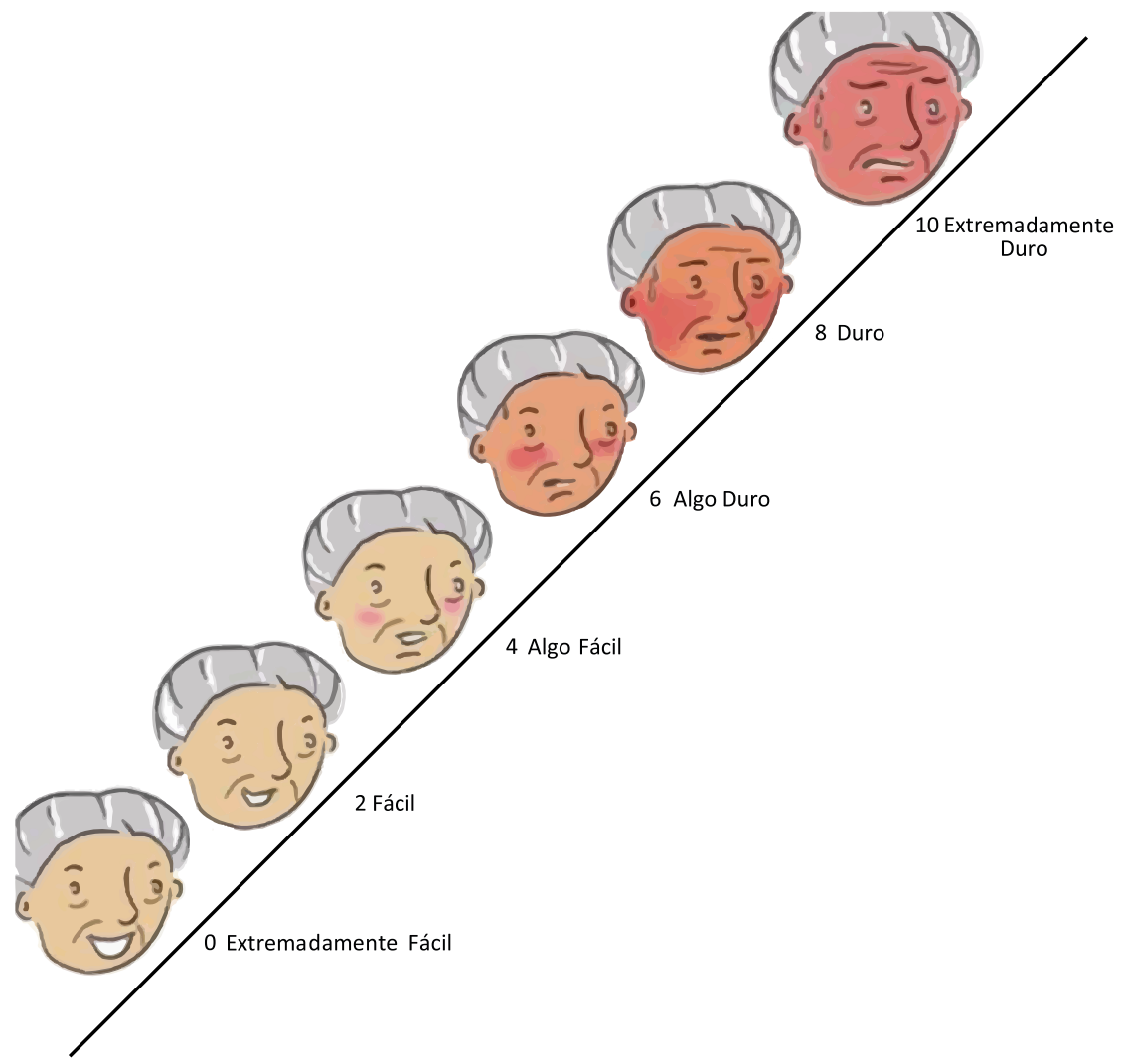

Escala de Borg

Fuente: Imagen tomada de la página web G-SE. Guillermo Peña García-Orea. Instituto Internacional de Ciencias del Ejercicio Físico y Salud. Disponible en https://g-se.com/ si-las-escalas-de-percepcion-de-esfuerzo-son-fiables-por-que-no-las-utilizamos-bpq57cfb26d3588c 
A continuación se propone una serie de ejercicios para las diferentes capacidades motrices y segmentos corporales, de los cuales se pueden escoger los que se desean trabajar en cada sesión. Al final del capítulo se presenta un cuadro con la secuencia del programa de ejercicios diseñada para 12 sesiones (1 mes).

\section{EJERCICIOS PARA MEJORAR FLEXIBILIDAD}

Cada ejercicio debe repetirse entre 8 y 10 veces

\section{Ejercicio No. 1.1}

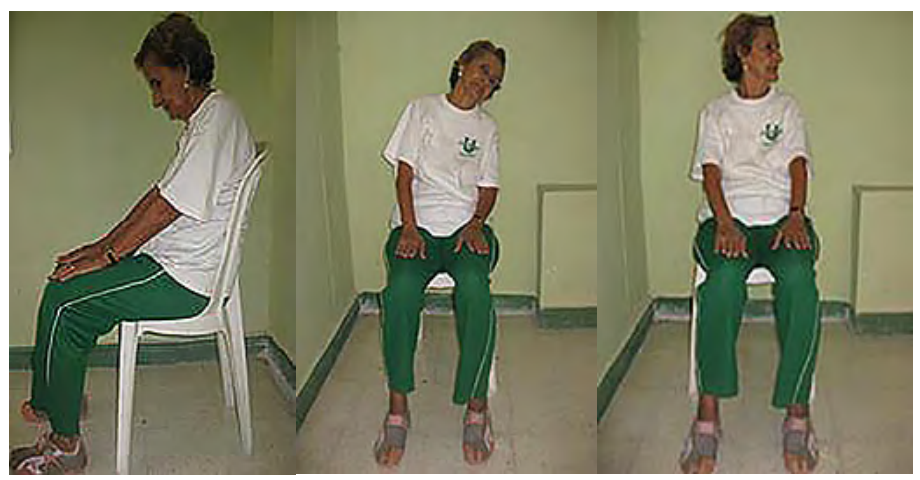

Flexión de cuello: En este ejercicio la persona debe estar sentada con la espalda recta. En esta posición dirige su cabeza hacia abajo tratando de mirar sus piernas, el movimiento finaliza regresando la cabeza a la posición inicial.

Inclinación de cuello: en la misma posición, la persona debe inclinar la cabeza hacia el hombro izquierdo y derecho alternadamente regresando finalmente a la posición inicial. 
Rotación de cuello: el ejercicio consiste en girar la cabeza hacia el lado izquierdo y derecho de manera alterna regresando a la posición inicial para finalizar el ejercicio.

\section{Ejercicio No. 1.2}

Miembros superiores:

Junte las manos en posición de orar. Lentamente levante los codos, de manera que los brazos queden paralelos al suelo, manteniendo las manos extendidas una contra la otra. Manténgase en esta posición por 10 a 30 segundos.

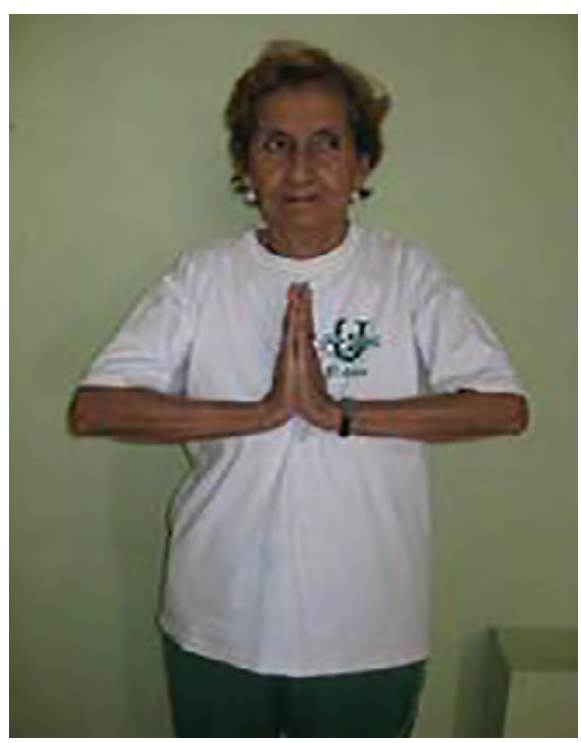




\section{Ejercicio No. 1.3}

1. Elevación de hombros alternativamente. Repetir 10 veces.

2. Elevación de hombros simultáneamente. Repetir 10 veces

3. Círculos de hombros de delante a atrás, y luego al revés. Repetir 8-10 veces cada uno.

4. Caminando, realizar movimientos de brazos hacia delante $y$ atrás de forma alternativa hasta la horizontal. Repetir 8-10 veces.

5. Rotaciones de brazos muy amplios hacia delante-atrás. Repetir 6-8 veces.

6. Realizar círculos por delante del tronco, con los brazos paralelos. Repetir 7 veces hacia delante, y 7 veces hacia atrás.

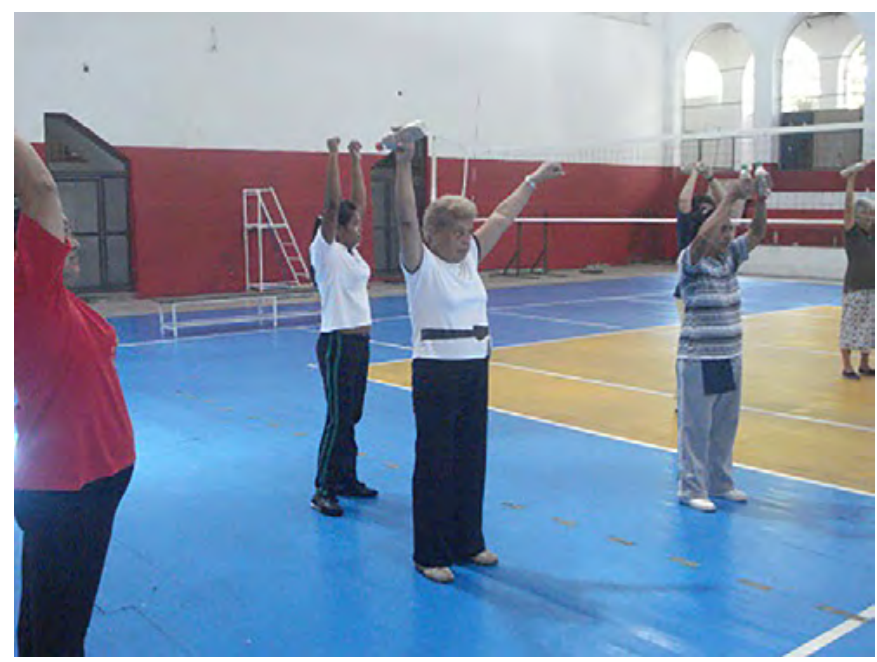




\section{Ejercicio No. 1.4}

1. Acuéstese en el piso, con una almohada bajo la cabeza.

2. Estire los brazos a los lados.

3. Doble los codos de manera que sus brazos formen un ángulo recto hacia abajo.

4. Manténgase en esta posición.

5. Doble los codos de manera que sus brazos formen un ángulo recto hacia arriba.

6. Manténgase en esta posición.

7. Mantenga los hombros pegados al suelo durantetodo el ejercicio.
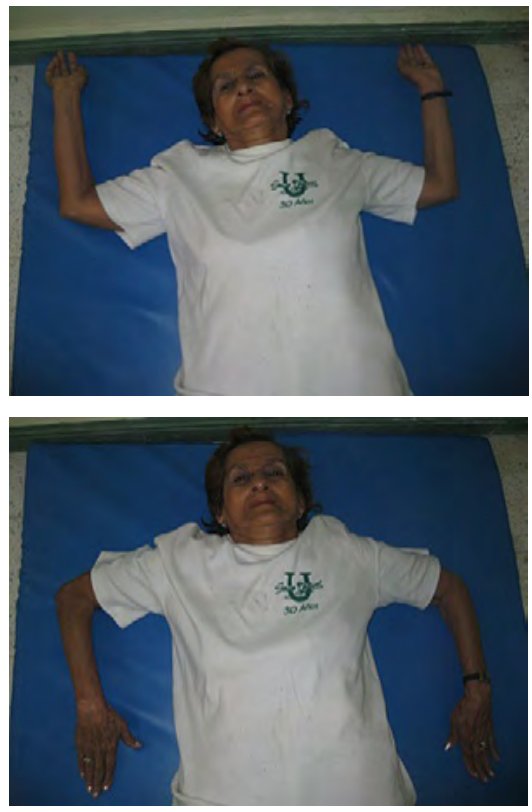


\section{Ejercicio No. 15}

1. Sostenga una toalla con su mano derecha.

2. Levante y doble su brazo derecho de manera de deslizar la toalla por la espalda.

3. Agarre el extremo de la toalla con su mano izquierda.

4. Suba la mano izquierda progresivamente con la toalla, la que también empuja su brazo derecho hacia abajo.

5. Cambie posiciones.

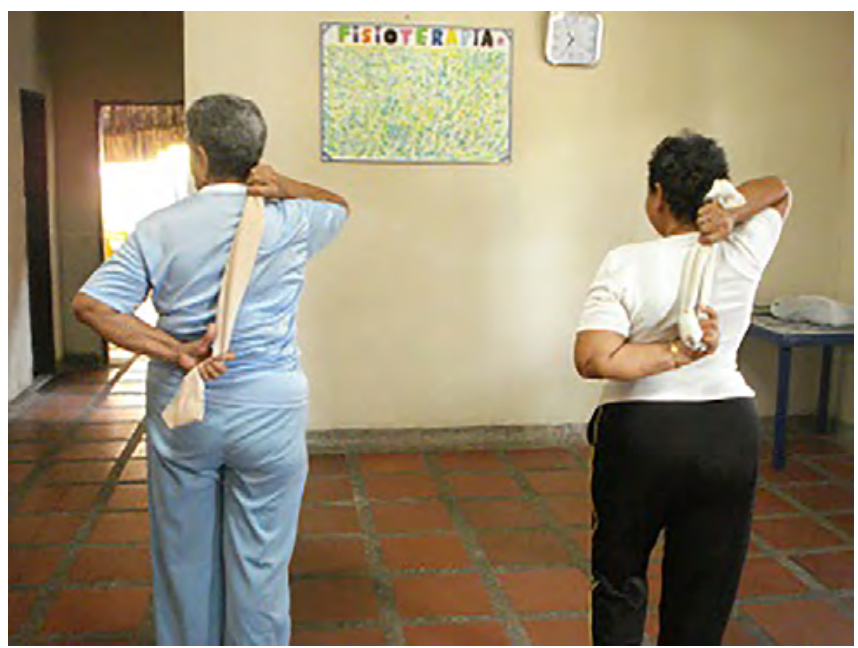


Ejercicio No. 1.6

Tronco:

Hacer movimientos de rotación completa del tronco hacia un lado y hacia el otro con acompañamiento de brazos en el mismo sentido, y de forma alternativa. Repetir 4 veces para cada lado.

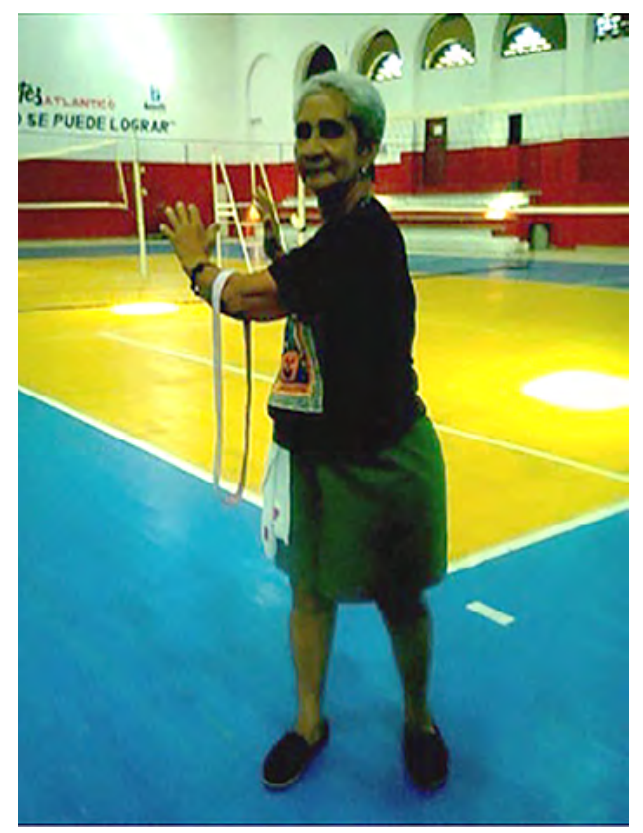




\section{Ejercicio No. 1.7}

Miembros inferiores:

1. Párese detrás de una silla, sosteniendo el respaldo con sus dos manos.

2. Inclínese hacia adelante desde las caderas, manteniendo la espalda y hombros derechos todo el tiempo.

3. Cuando la parte superior del cuerpo esté paralela al piso, manténgase en esta posición.

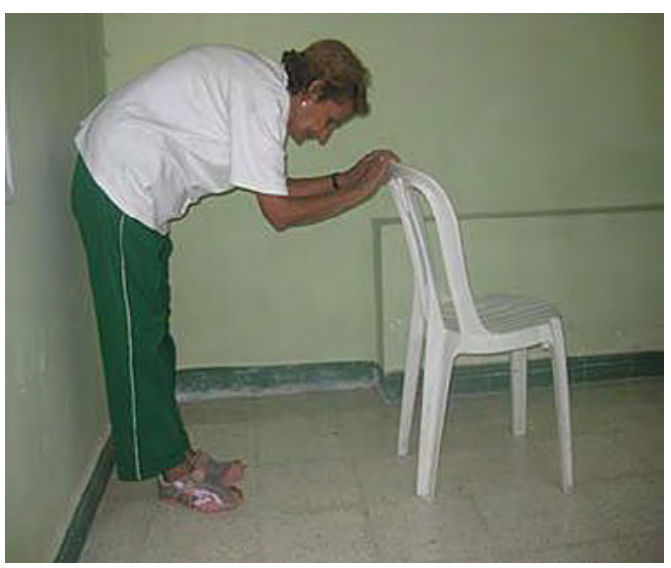


Ejercicio No. 1.8

1. Siéntese en una silla.

2. Estire las piernas de frente a usted.

3. Doble los tobillos de manera que los pies miren a usted.

4. Doble los tobillos de manera que los pies se alejen de usted.

5. Si no siente el tirón, repita con los pies levemente levantados del suelo.

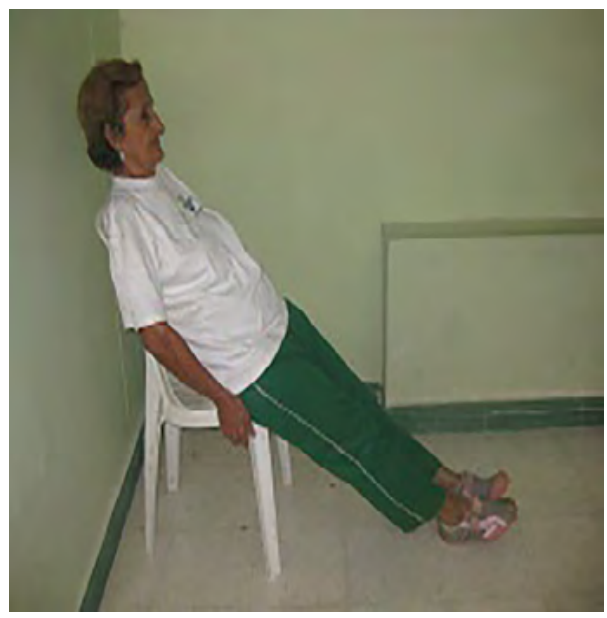




\section{Ejercicio No. 1.9}

1. Siéntese de lado en un banco o con una silla enfrente.

2. Mantenga una pierna estirada, sin flexionar la rodilla, en el banco.

3. Mantenga la otra pierna fuera del banco, con la planta del pie apoyada en el suelo.

4. Enderece la espalda.

5. Inclínese hacia adelante desde las caderas (no de la cintura) hasta que sienta el estiramiento en la pierna sobre el banco, manteniendo espalda y hombros derechos. Para el caso de las personas con prótesis de cadera, omitir este ejercicio, a no ser que se lo autorice su médico.

6. Manténgase en esta posición.

7. Repita con la otra pierna.

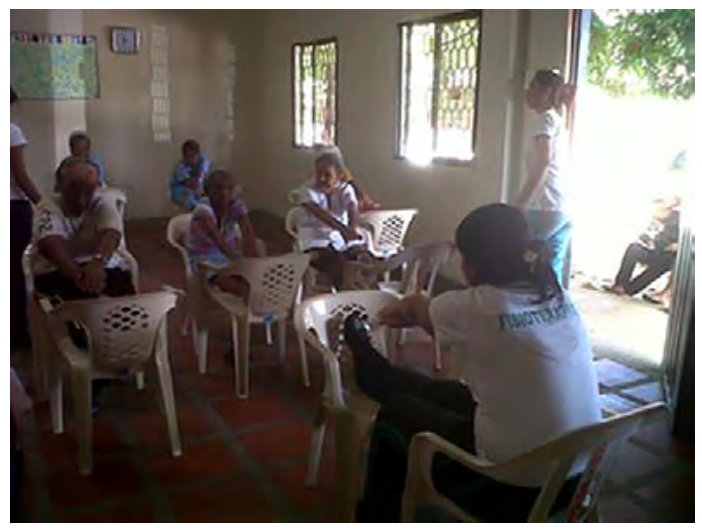


Ejercicio No. 1.10

1. Pararse con las manos contra la pared, los brazos extendidos.

2. De un paso atrás con una pierna, talón y pie sobre el suelo.

3. Manténgase en esta posición.

4. Repita con la otra pierna.
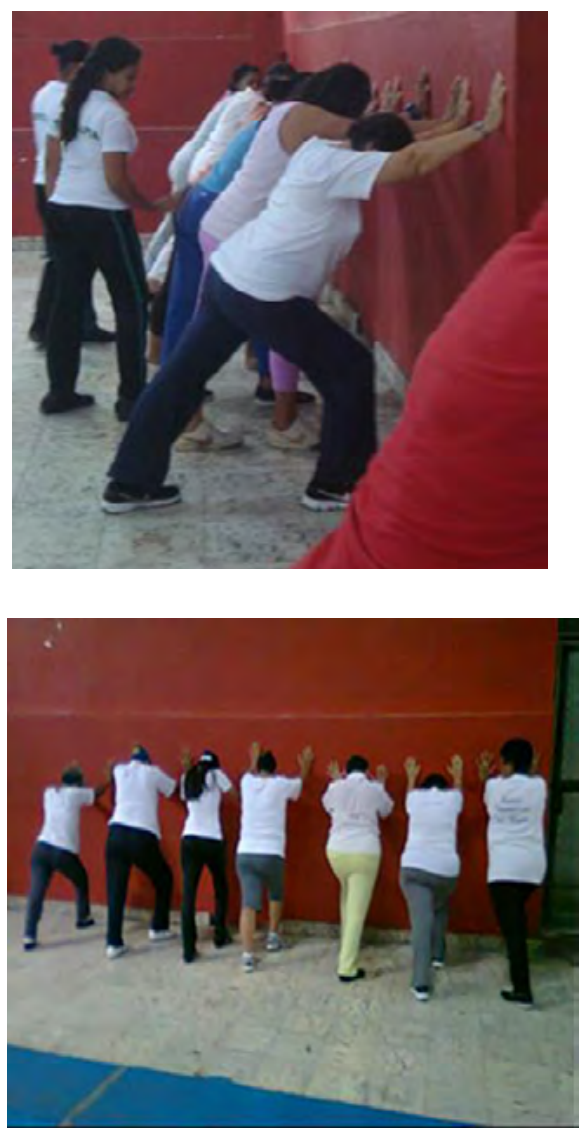


\section{Ejercicio No. 1.11}

1. No realizar este ejercicio en caso de tener una prótesis de cadera, a no ser que el médico lo autorice.

2. Acostado de espaldas en el suelo, con las rodillas flexionadas y los pies extendidos sobre el suelo.

3. Mantener los hombros pegados al suelo durante todo el tiempo.

4. Manteniendo las rodillas juntas, bajar las piernas hacia un lado, sin forzarlas.

5. Manténgase en esta posición durante 10 a 30 segundos

6. Llevar las rodillas al centro y repetir el ejercicio hacia el otro lado. Se realiza de 3 a 5 veces en cada lado.
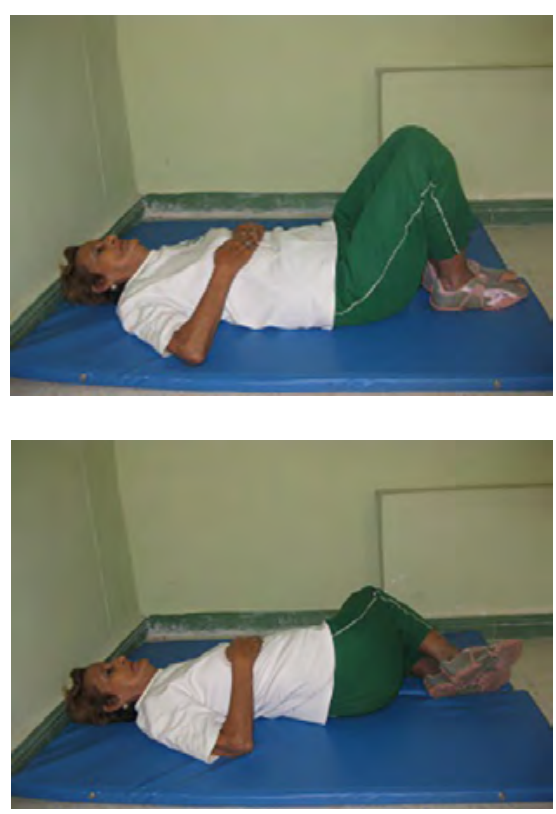


\section{Ejercicio No. 1.12}

1. Acuéstese de lado, apoye la cabeza sobre una almohada o mano.

2. Doble la rodilla que está arriba, agarre el talón de esa pierna.

3. Tire suavemente esa pierna hasta que la cara anterior del muslo se estire.

4. Permanezca en esa posición.

5. Cambie de lado y repita el ejercicio.

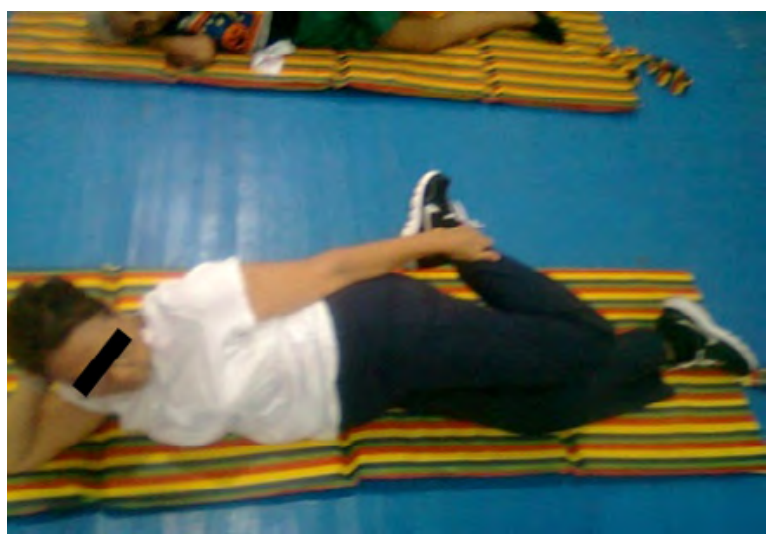




\section{Ejercicio No. 1.13}

1. Acostada boca arriba con las piernas flexionadas. Extender una pierna tomando la pantorrilla con ambas manos.

2. Tratar de llevar la pierna hacia la cara hasta que se sienta tensión en los músculos de atrás de muslo y pierna, sin que provoque dolor. Repetir con la pierna contraria.

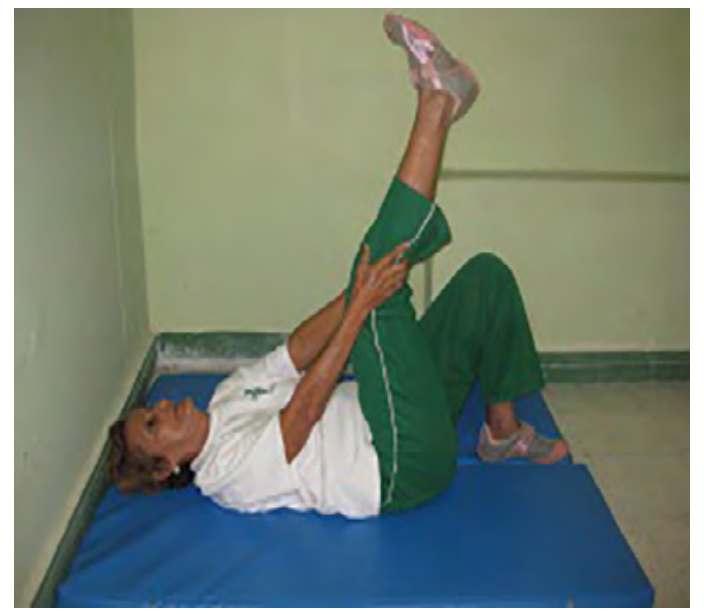

100

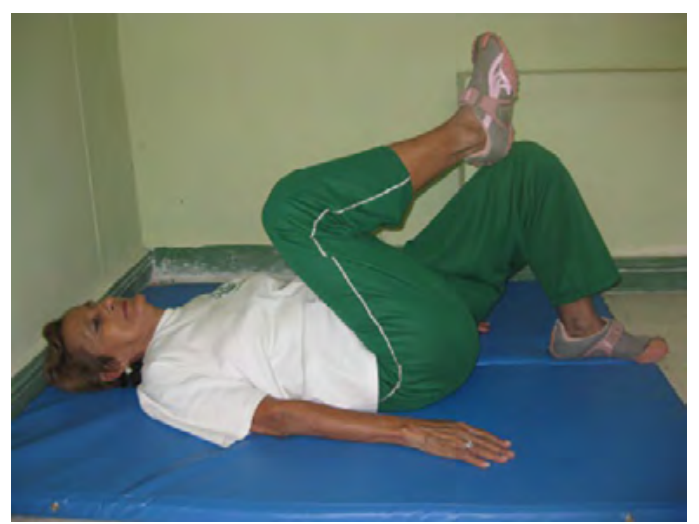




\section{EJERCICIOS PARA MEJORAR LA COORDINACIÓN}

\section{Ejercicio No. 2.1}

Nombre: "De atrás adelante".

Objetivos: Mejorar la coordinación.

Organización: Se dividirán en dos equipos y se formarán en hileras.

Desarrollo: A la voz de mando, los participantes pasarán el balón de atrás hacia delante. Se entregará con la mano izquierda y se recibe con la derecha extendiendo la mano hacia atrás. Tan pronto el balón llegue al usuario que ocupa el primer puesto, este lo sostendrá en las manos, camina con él hacia el último puesto de la fila y comenzará a pasarlo nuevamente como al inicio. Cada vez que el balón llegue al primer puesto el que ocupa dicho lugar caminará con él hacia el último puesto, de su equipo y así sucesivamente se desarrollará el juego hasta que los usuarios adopten la posición inicial. El equipo que primero se ubique en esta posición será el ganador.

\section{Ejercicio No. 2.2}

Nombre: "Mar y tierra".

Objetivos: Mejorar la coordinación.

Organización: Formados en filas, detrás de una línea que representa el límite.

Desarrollo: A la voz de mando, que puede ser mar o tierra, el jugador sacará una pierna al frente según cuál sea la orden (pierna derecha: mar; pierna izquierda: tierra). 


\section{Ejercicio No. 2.3}

Nombre: "El acróbata".

Objetivos: Mejorar el equilibrio y la coordinación.

Organización: Se forman dos equipos en hileras detrás de una línea de salida. Delante de cada equipo se traza una línea que simula una cuerda floja.

Desarrollo: A la señal, los primeros participantes de cada equipo saldrán caminando por encima de la cuerda floja; al final de esta realizarán un ejercicio sencillo de brazos y piernas. Gana el equipo que más puntos haya acumulado.

\section{Ejercicio 2.4}

Nombre: "El Robot".

Objetivos: Mejorar la coordinación.

Organización: se organizan las personas en un círculo o en dos filas.

La persona inicia la actividad de pie con los brazos adosados al $|102|$ cuerpo (posición firme).

1. Brazo izquierdo adosado al cuerpo, brazo derecho arriba, pierna izquierda un paso al frente y lo deja apoyado.

2. Posición inicial.

3. Brazo derecho adosado al cuerpo, brazo izquierdo arriba, pierna derecha da un paso al frente y la deja apoyada.

4. Regrese a la posición inicial. 


\section{Ejercicio No. 2.5}

Nombre: "El Túnel".

Objetivos: Mejorar la coordinación.

Organización: Se formarán equipos en hileras con las piernas separadas.

Desarrollo: A la señal, comenzarán a pasar la pelota por encima de la cabeza, al llegar al último jugador este se incorpora al inicio de la hilera. Gana el equipo que primero termine.

Regla: No se puede dejar caer la pelota. El último usuario con la pelota debe incorporarse al inicio de la hilera.

\section{Ejercicio No. 2.6}

Nombre: "Camina en Zigzag".

Objetivos: Mejorar la coordinación.

Organización: Formarán equipos en hileras, a una distancia de un metro del primer jugador; se colocan tres obstáculos.

Desarrollo: A la voz de mando los primeros participantes de cada equipo saldrán caminando entre los obstáculos hasta llegar al último, regresan por fuera dándole la salida a su compañero e incorporándose al final de la formación. 


\section{Ejercicio No.2.7.}

Nombre: "Pasar al río".

Objetivos: Mejorar la coordinación y el equilibrio.

Organización: Se forman equipos en hileras detrás de una línea de salida; a una distancia de 5 a 8 metros de esta se traza otra de llegada. Delante de cada equipo se colocan pequeñas tablitas (que simulan las piedras) separadas entre sí a una distancia de $60 \mathrm{~cm}$. que representan el río.

Desarrollo: A la señal, el primer participante de cada equipo pasa por el río tratando de no caer en el agua y en equilibrio sobre las tablillas de madera. Una vez rebasada la línea de llegada sale el siguiente jugador. Cada equipo forma su hilera detrás de la línea de llegada. Gana el equipo que primero termine.

Regla: No deben salir hasta que el jugador no haya cruzado la línea de llegada. 


\section{Ejercicio No. 2.8}

Nombre: "Relevo de parejas".

Objetivos: Mejorar la coordinación.

Organización: Se forman equipos en hileras y por parejas detrás de una línea de salida. Frente a cada equipo, y a una distancia determinada por el profesor, se coloca una banderita.

Desarrollo: A la señal de mando, la primera pareja de participantes de cada equipo sale y camina hacia la banderita tomados de las manos. Cada vez que el organizador dé una palmada, las parejas que caminan dan una vuelta en el lugar y a continuación, caminando para dar un rodeo a la banderita, regresan. Al llegar a la línea de salida se colocan al final de su equipo y la pareja que se encuentra esperando sale a realizar la misma actividad y así sucesivamente, hasta que todos los equipos finalicen. Gana el equipo que primero termine.

\section{Ejercicio 2.9}

\section{Ejercicios con pelota}

Pasar la pelota por detrás de la cabeza, alrededor de la cintura y por detrás de cada rodilla.
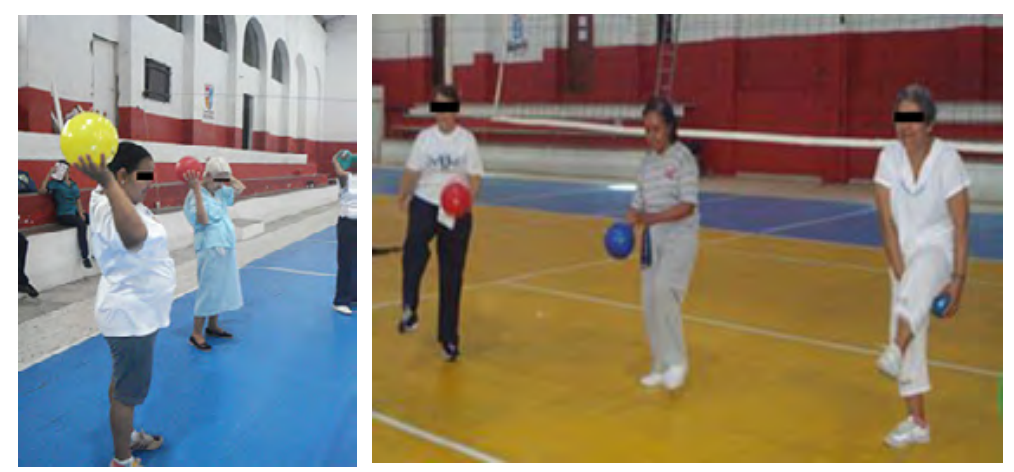


\section{Ejercicio 2.10}

\section{Grupal o en pareja.}

Se forma un círculo con los compañeros de grupo. Se coloca una persona en el centro del círculo. La persona debe estar con los ojos cerrados. Al azar, uno de los compañeros hará un sonido, ya sea aplaudiendo, con un silbato o alguna otra forma. La persona del centro debe señalar quién de los compañeros hizo el sonido.

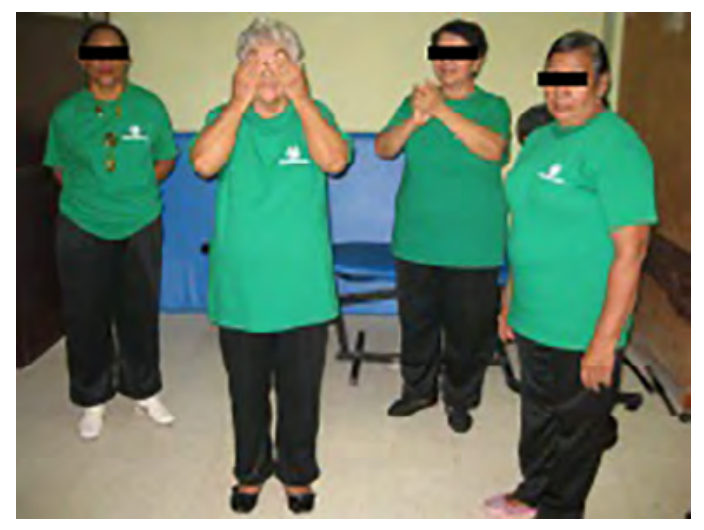

$|106|$

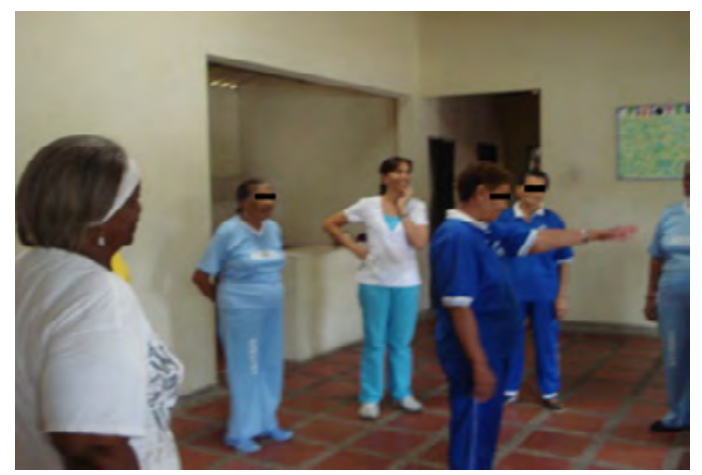




\section{Ejercicio 2.11}

\section{Coordinación visomotora}

Se coloca una persona frente a otra. Se le explica que, sin hablar, su compañera le señalará con un dedo la parte de su cara que se debe tocar (el oído, frente, la nariz, etc.). Al principio lo hará despacio, aumentado progresivamente la velocidad, para observar el grado de respuesta.
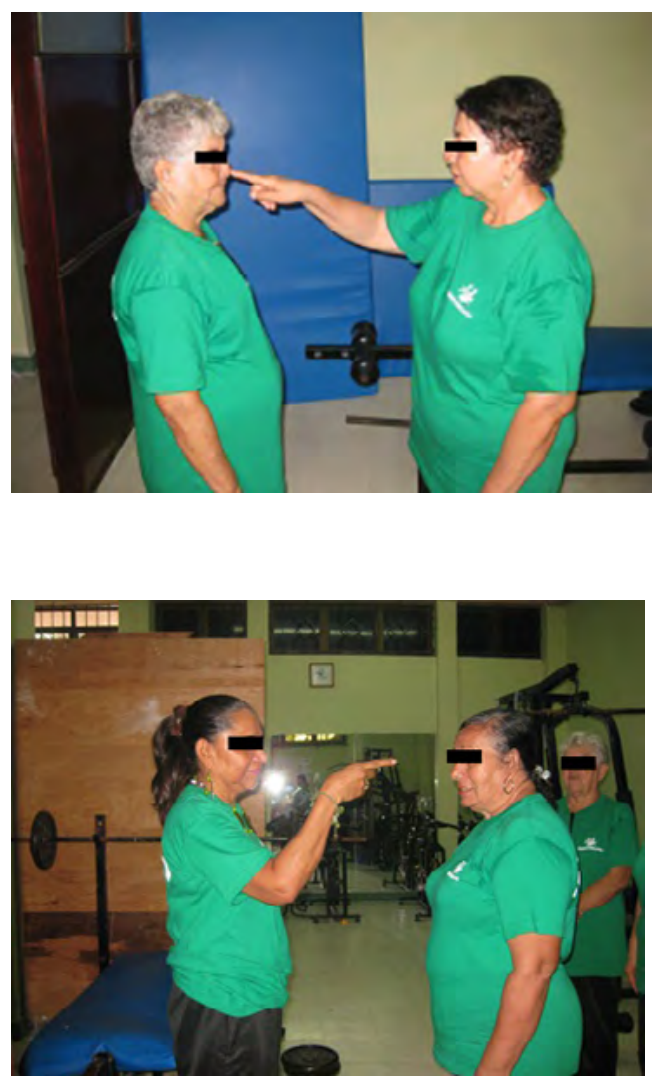


\section{Ejercicio 2.12}

Nombre: "El tren".

Objetivo: Mejorar la coordinación.

Materiales: Sillas.

Organización: Sentados en sillas formando dos filas, una en frente de la otra.

Desarrollo: El fisioterapeuta permanece de pie y anuncia ser el revisor del vagón. Señala el lugar de entrada y salida de los pasajeros del vagón.

Cuando el revisor nombra una estación de tren los participantes deberán cambiarse de lugar. Cuando dé la orden de salida todos desalojarán el tren por la zona indicada, y para entrar, de nuevo darán la vuelta por la parte exterior. Mientras, el revisor se sentará en una silla, quedando un viajero sin ella, pasando a ser el nuevo revisor.

Reglas: No se debe salir o entrar por cualquier zona que no sean las $\mid 108$ establecidas. 


\section{Ejercicio 2.13}

Nombre: "Bolos".

Objetivo: Mejorar la coordinación.

Material: Un juego de bolos de madera u otro material según posibilidades.

Organización: El lanzador se coloca a una distancia de 10 m. (Línea de lanzamiento).

Desarrollo: El primer jugador lanza la bola intentando tirar el máximo número de bolos; dispondrá de dos lanzamientos en cada tanda para tirarlos todos. Anotará el número de bolos tirados y cederá el turno a su compañero.

Reglas: Gana el jugador que en 5 rondas obtenga el mayor puntaje.
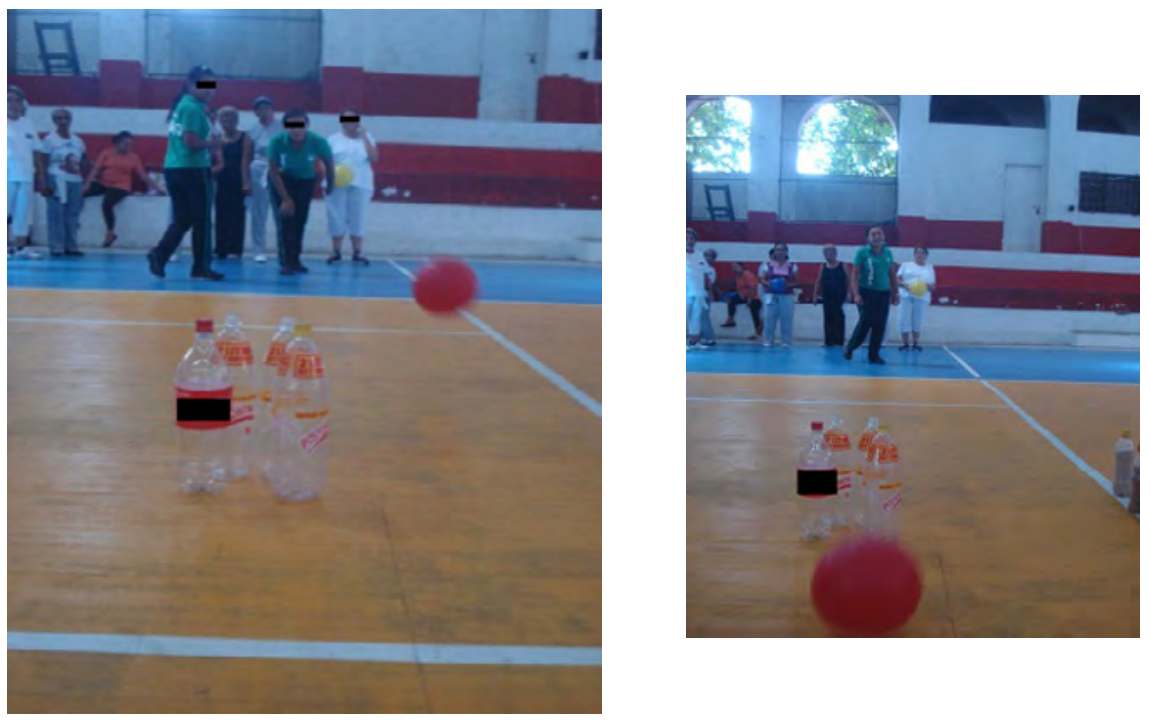


\section{EJERCICIOS PARA MEJORAR EL EQUILIBRIO}

\section{Ejercicio No. 3.1}

Posición: Sentado(a) en una silla sin brazos, separa la espalda del espaldar y las piernas ligeramente abiertas a la anchura de las caderas. Cruza los brazos al frente del pecho separados del cuerpo. Levántese de la silla sin abrir los brazos y solo con la ayuda de las piernas. Siéntese de la misma forma sin inclinar la espalda hacia adelante. Tres series de 8 o 10 repeticiones.

\section{Ejercicio No. 3.2}

Posición: De pie. Mire hacia un punto fijo. Manos a la cintura. Eleve la rodilla derecha en posición de $90^{\circ}$ Mantenga por 20 segundos y baje. Alterne con la izquierda. Repita cinco veces por pierna.

\section{Ejercicio No. 3.3}

Posición: De pie. Mire hacia un punto fijo. Brazos abiertos y extendidos a los lados, como en forma de T. Despegue el pie del piso y estire al frente la pierna derecha en posición recta. Mantenga por 20 segundos y baje. Alterne con la izquierda. Repita esto tres veces por pierna.

\section{Ejercicio No. 3.4}

Posición: De pie. Mire hacia un punto fijo. Brazos extendidos al frente. Eleve la pierna derecha estirada hacia un lado del cuerpo (lateralmente). Mantenga por 20 segundos y baje. Alterne con la pierna izquierda. Repita esto tres veces por pierna. 


\section{Ejercicio No. 3.5}

Posición: De pie. Mire hacia un punto fijo. Brazos arriba. Doble para atrás la rodilla derecha en posición de $90^{\circ}$ Mantenga por 15 segundos y relaje los brazos. Alterne con la otra pierna. Repita tres veces por pierna.

\section{Ejercicio No. 3.6}

Balance de pie. Párese con las piernas abiertas a la anchura de las caderas, hombros relajados, espalda recta, mirada al frente, brazos a los lados. Levante ligeramente del suelo cada pie, balanceándose de lado a lado; de manera que en cada balanceo, el peso de su cuerpo repose en un solo pie a la vez.

\section{Ejercicio No. 3.7}

Este se puede hacer solo(a) o en compañía, uno frente al otro. Posición: De pie, estire los brazos a los lados, sobre una superficie recta, o una línea pintada en el piso párese en frente de su compañero(a) a una distancia de 3 metros entre los dos y de un paso seguido del otro pie (no se pueden dejar espacios entre cada paso) hasta que se encuentren los dos.

\section{Ejercicio No. 3.8}

De pie. Separe las piernas al ancho de las caderas. Coloque los brazos como haciendo un círculo con ellos y las palmas mirando al pecho, como si estuviera abrazando un árbol. Flexione las piernas levemente e intente enderezar la columna. Respire inhalando por nariz y exhalando por boca. Mantenga esta posición al menos 20 segundos. 


\section{Ejercicio No. 3.9}

De pie. Piernas juntas. Extienda los brazos al frente. Incline el tronco hacia adelante al tiempo que eleva por detrás una pierna. Baje hasta donde pueda conservar el equilibrio, respire tres veces y regrese para hacerlo con la otra pierna.

\section{Ejercicio No. 3.10}

Paso alto. En un escalón, párese sobre el pie derecho, estire los brazos al frente y extienda la pierna izquierda hacia atrás, dejando el pie ligeramente despegado del cajón a unos $10 \mathrm{~cm}$. Todo el peso de su cuerpo debe estar suspendido en un solo pie sobre el cajón. Mantenga la espalda recta y la mirada al frente. Por 20 segundos y alterne con la otra pierna. Repita esto tres veces. Hacer tres veces por semana.

\section{Ejercicio 3.11}

Equilibrio estático, 4 repeticiones:

De pie, con los talones y tobillos unidos y la punta de los pies hacia fuera, brazos laterales (mantener la posición).

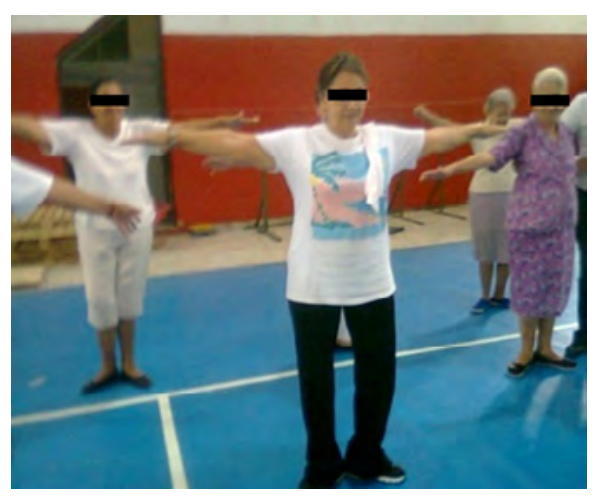




\section{Ejercicio 3.12}

Equilibrio dinámico, 4 repeticiones:

Camine tobillo-con-dedo. Coloque su tobillo justo en frente de los dedos del pie opuesto cada vez que dé un paso. Su tobillo y dedos deben tocarse o casi tocarse.

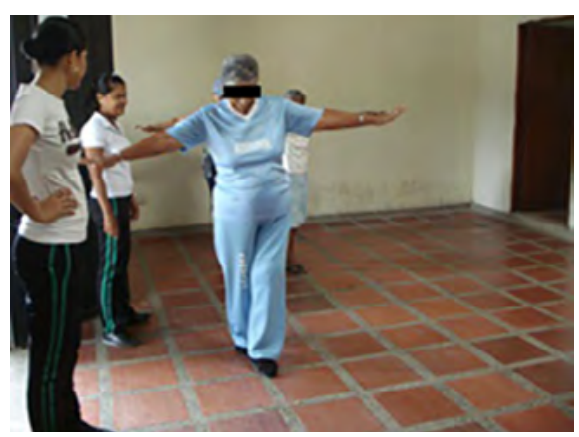

\section{Ejercicio 3.13}

Equilibrio dinámico, 4 repeticiones:

De pie. Tobillos separados $10 \mathrm{~cm}$. Brazos a lo largo del cuerpo. Inclinar el cuerpo hacia delante y hacia atrás apoyando el peso en los dedos de los pies sin elevar los talones.

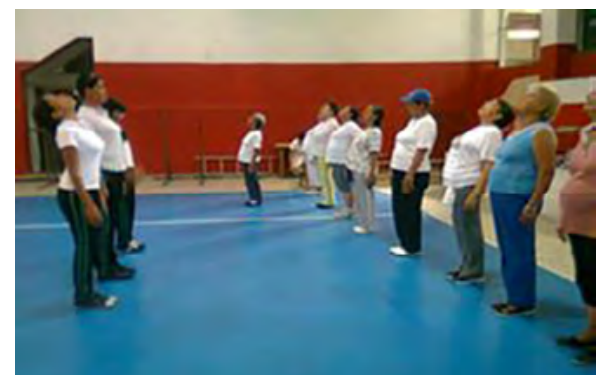




\section{Ejercicio 3.14}

Equilibrio estático, 4 repeticiones:

De pie, con los talones y tobillos unidos y la punta de los pies hacia fuera, brazos laterales (mantener la posición).

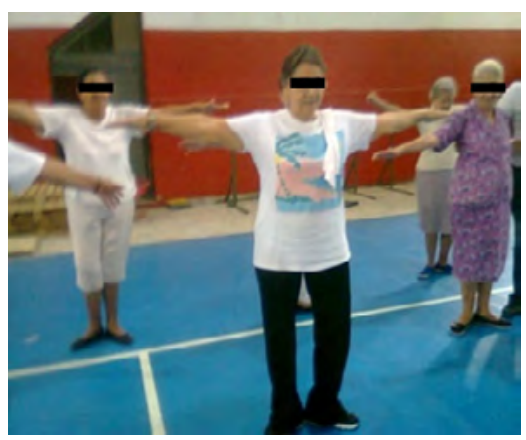

\section{Ejercicio 3.15}

Equilibrio estático, 4 repeticiones:

De pie, con los pies a la anchura de los hombros, realizar giros completos; primero por el lado derecho y luego por el lado izquierdo. 


\section{Ejercicio 3.16}

Equilibrio dinámico, 4 repeticiones:

Párese en un pie (puede hacerse mientras espera en fila o en un paradero de bus). Alterne el pie. Párese y siéntese sin usar sus manos

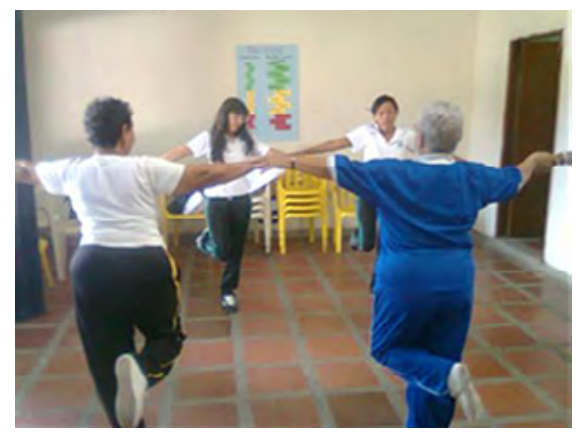

\section{Ejercicio 3.17}

Equilibrio estático, 4 ejercicios:

De pie, con los pies a la anchura de los hombros, el rehabilitador empuja suavemente la cabeza del paciente, hacia delante, lateral derecho e izquierdo y hacia atrás.
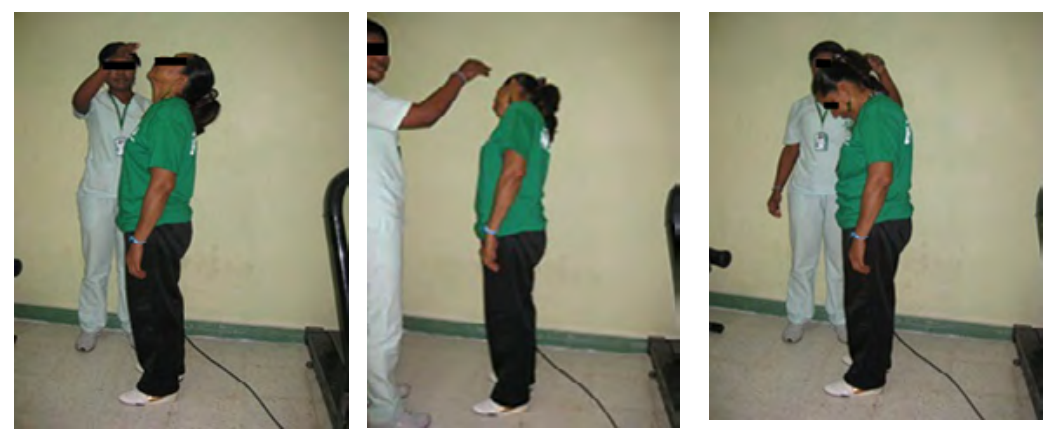


\section{EJERCICIOS DE RESISTENCIA AERÓBICA}

\section{Ejercicio 4.1}

¡De caminata a marcha!

La caminata es el ejercicio más básico cuando de mejorar la resistencia aeróbica se trata, ya que es una habilidad motora que necesita de un aprendizaje específico. Todo el mundo sabe caminar si se encuentra en condiciones normales. Se cambia el concepto de caminata por el de marcha, ya que en vez de caminar como si se estuviera paseando, los adultos mayores van a marchar a un ritmo vigoroso, según su tolerancia.

- Actividad principal: Marchar a un ritmo intenso, acompañado de balanceo enérgico de los brazos, llevándolos a semi-flexión, y una amplitud mayor de la caminata normal.

- Duración: Mínimo 20 minutos para asegurar el funcionamiento del sistema aeróbico.

- Frecuencia: 2 veces por semana.

- Al final se realiza un estiramiento suave para la recuperación y relajación. 


\section{Ejercicio 4.2}

Rutina de aeróbicos:

- Toma de tensión arterial.

- Se comienza con ejercicios para manejar la respiración y adecuar el sistema respiratorio al ejercicio.

- Luego se realiza un calentamiento para preparar las articulaciones, músculos y otras partes del organismo al ejercicio.

- Se lleva a cabo la actividad principal que es la RUTINA DE AERÓBICOS.

- Se termina con ejercicios de relajación y se toma la tensión arterial.

\section{Ejercicio 4.3}

\section{Principales ejercicios de resistencia aeróbica}

Estos ejercicios hacen participar a un gran número de músculos del organismo:

- Andar o correr. Caminar es uno de los mejores ejercicios, especialmente a partir de cierta edad, porque puede hacerse en cualquier sitio; no requiere un equipamiento especial, es sencillo, puede hacerlo casi todo el mundo y permite ejercitar un gran número de músculos del organismo.

- Nadar, hacer bicicleta estática o pasear en bicicleta. Estas actividades son especialmente recomendables para las personas que tengan problemas en sus articulaciones, o problemas de obesidad. 


\section{EJERCICIOS DE FUERZA}

\section{Ejercicio No. $\mathbf{5 . 1}$}

Ejercicios de fuerza en miembros superiores, 4 repeticiones:

De pie, con los brazos al lado del cuerpo y con una mancuerna o botella de arena (1 1 o 2 libras) en cada mano, levantarlos hasta llevarlos por encima de la cabeza.

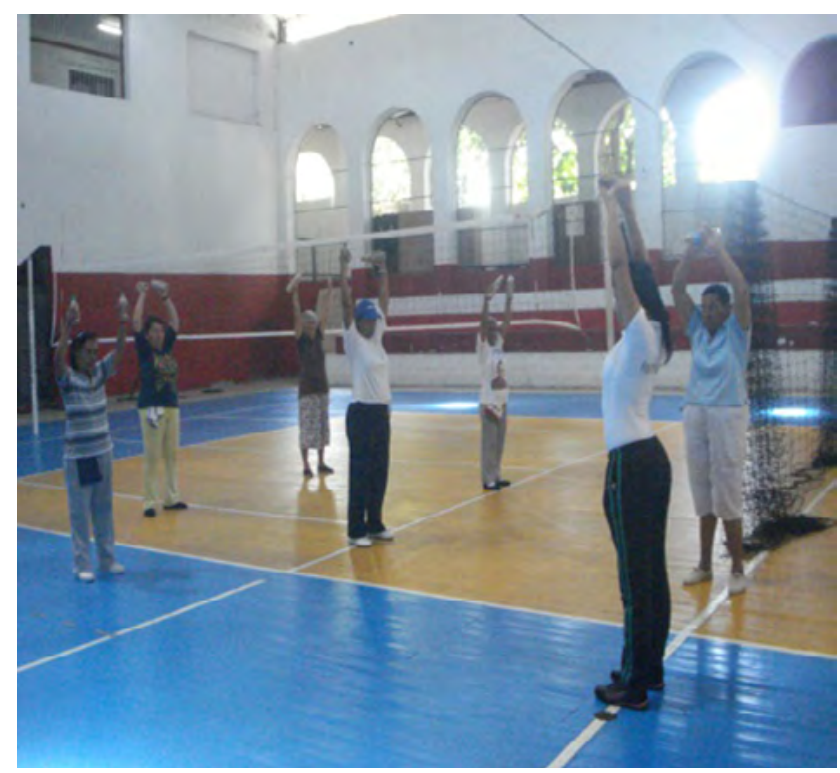




\section{Ejercicio No. 5.2}

Ejercicios de fuerza en miembros superiores, 4 repeticiones:

Sentado, con el codo apoyado en el muslo y con una mancuerna o botella de arena ( 1 o 2 libras), hacer flexiones y extensiones del antebrazo.
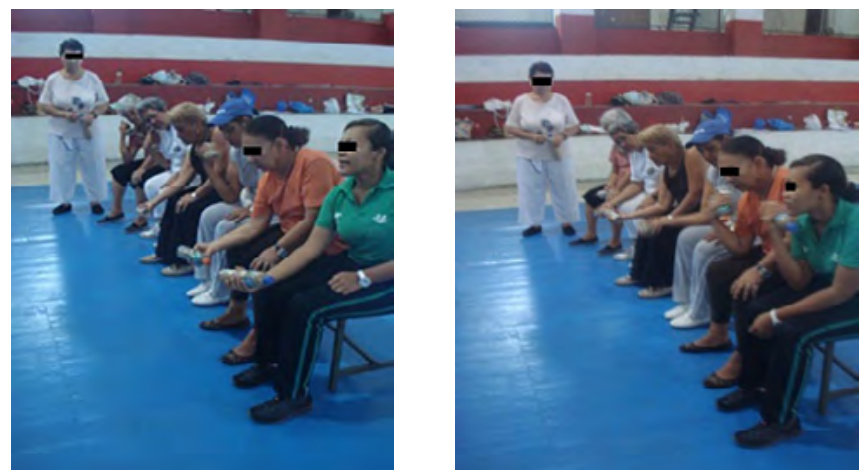

\section{Ejercicio No. 5.3}

De pie con mancuernas o botellas de arena (1 0 o 2 libras), hacer flexiones de los codos al frente de $45^{\circ}$ máximo. 4 repeticiones.

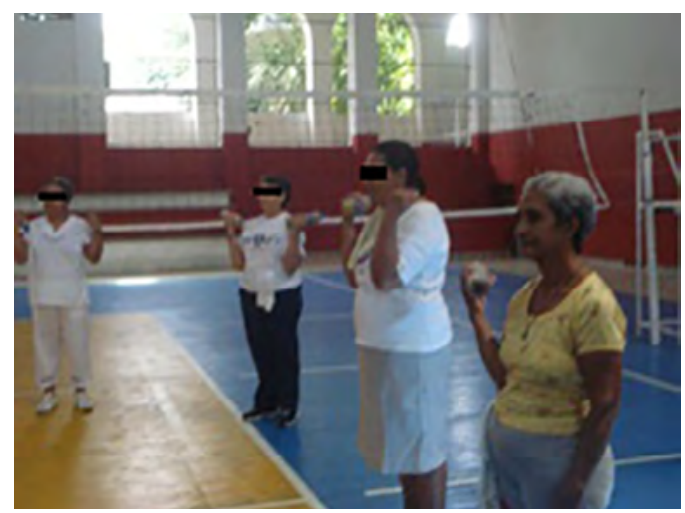




\section{Ejercicio No. 5.4}

Acostado boca arriba, con una mancuerna o botella de arena (1 o 2 libras), abrir los brazos en cruz, levantarlos lateralmente, hasta colocarlos frente a la cara.

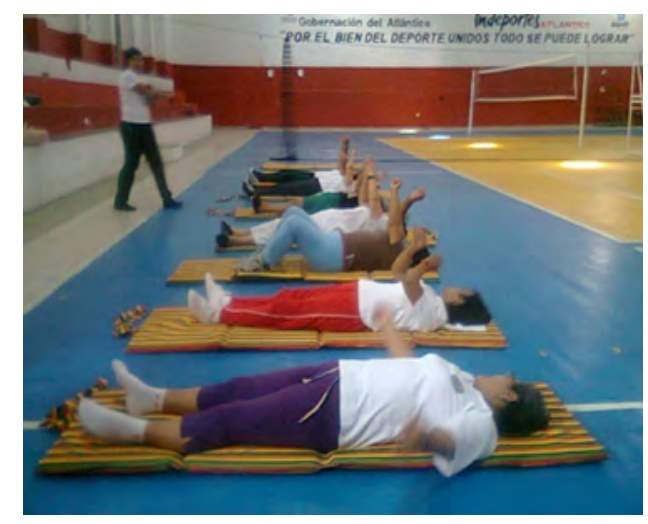

\section{Ejercicio No. 5.5}

Con ambas manos, con las palmas hacia abajo, se elevan los brazos al frente, estirando la liga lo más que pueda. Se efectúan de 5 a 10 repeticiones. Si se puede, trabajar primero un lado y luego el otro.

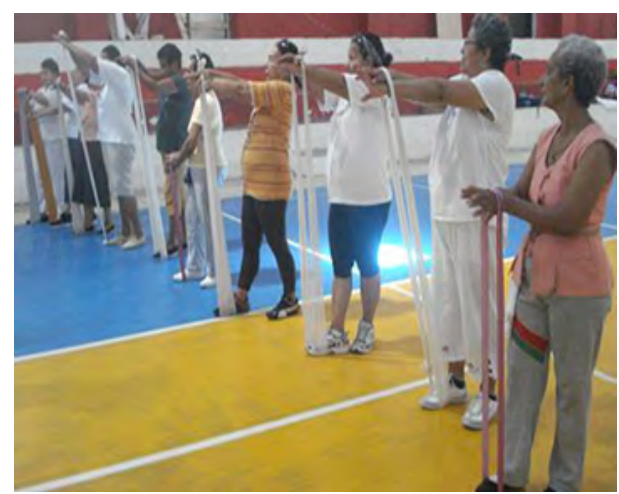




\section{Ejercicio No. 5.6}

De pie, levantar las manos frente al cuerpo, flexionando lateralmente los codos a $90^{\circ} \mathrm{y}$ las manos frente a los hombros.

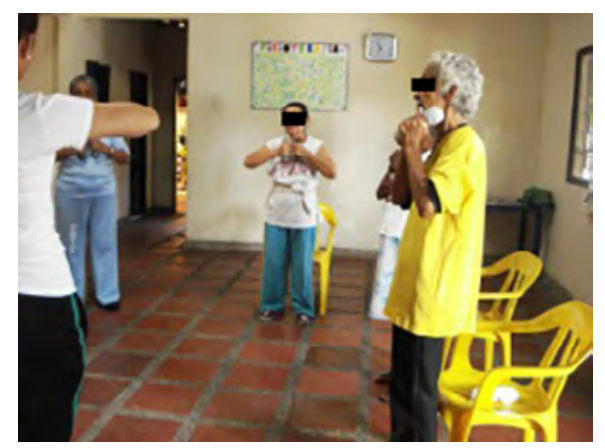

\section{Ejercicio No. 5.7}

Fortalecimiento de músculos del hombro y dorsales. El ejercicio se puede hacer sentado o de pie. Se sostiene la liga (Thera-band) con los pies. El extremo opuesto se toma con ambas manos con las palmas hacia abajo. Se estira la liga girando los codos hacia fuera, lo máximo que pueda. Se efectúan de 5 a 10 repeticiones.
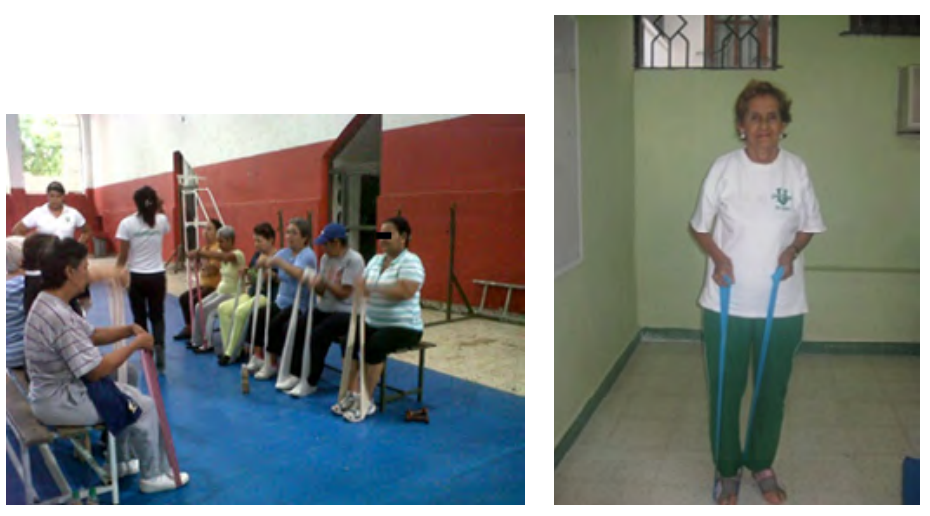


\section{Ejercicio No. 5.8}

Fortalecimiento de músculos posteriores del brazo (tríceps) y hombro. Con una mano se toma aproximadamente la liga a la mitad. Con la otra se toma el extremo distante de la liga. Se trata de estirar la liga a la máxima posición. Se realizan de 5 a 10 repeticiones.
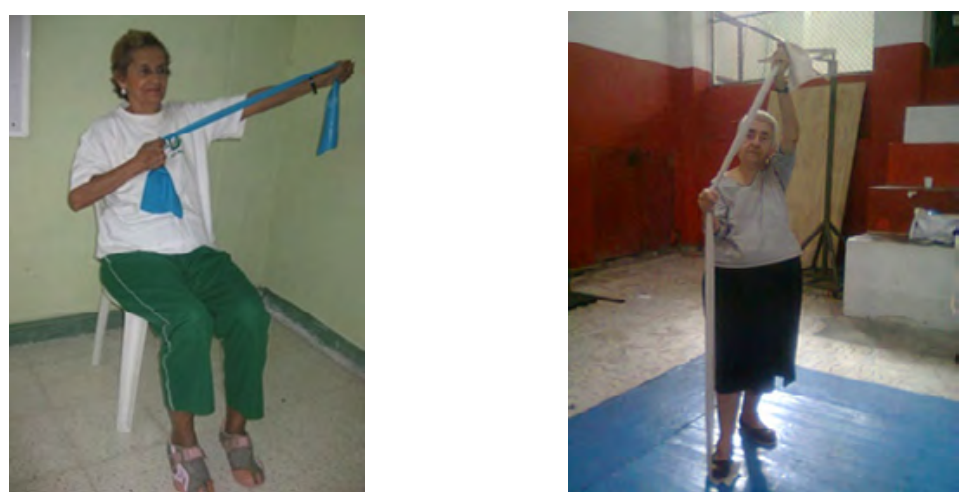

\section{Ejercicio No. 5.9}

Fortalecimiento de músculos dorsales y lumbares. De rodillas, con los brazos cruzados sobre el pecho. Hacer una flexión del tronco, tratando de tocar el tórax con la barbilla. Hacer 5 a 10 repeticiones.
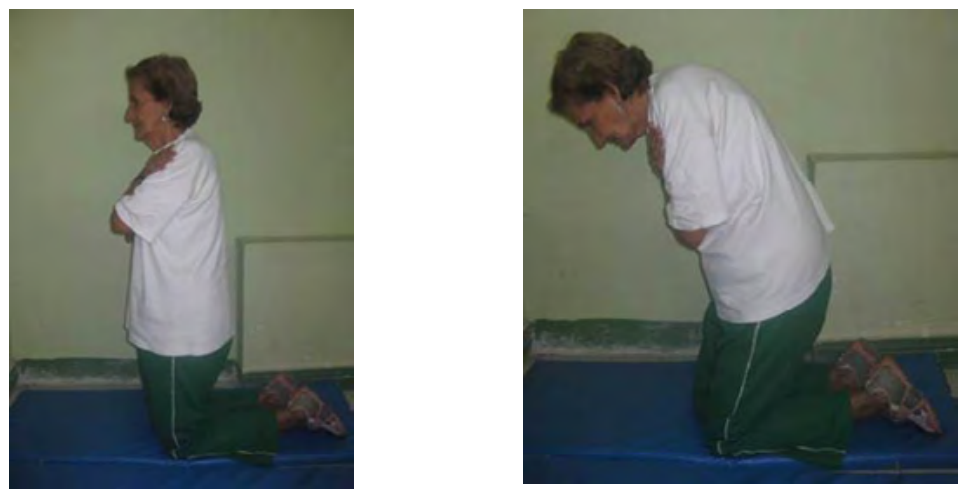


\section{Ejercicio No. 5.10}

Fortalecimiento de músculos dorsales y lumbares. Acostado, boca arriba con los brazos cruzados sobre el pecho flexionar el tronco, tratando de tocar el pecho con la barbilla. Hacer 5 a 10 repeticiones.
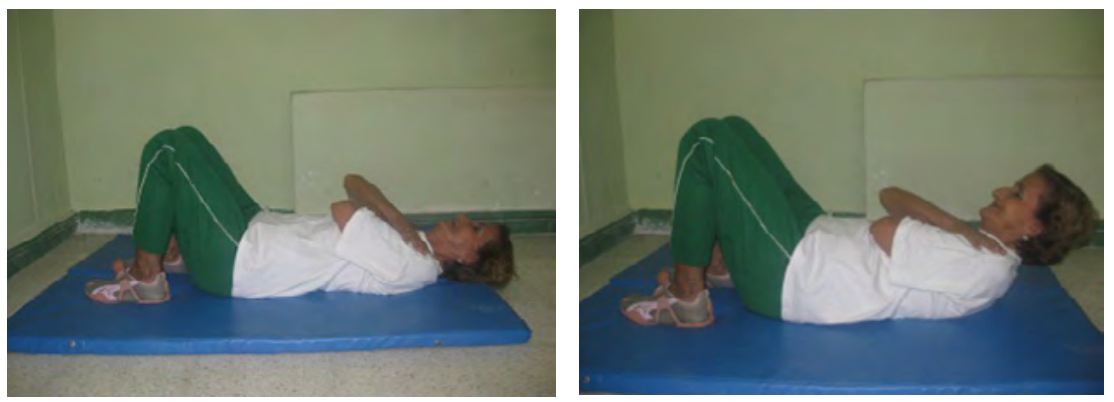

\section{Ejercicio No. 5.11}

Fortalecimiento de músculos anteriores de muslo y pies. Sentado en una silla ( $\sin$ descansabrazos) colocar las manos sobre las rodillas. Ponerse de pie utilizando solo la fuerza de las extremidades inferiores sin apoyarse con las manos sobre las piernas u otro objeto. Hacer de 5 a 10 repeticiones.
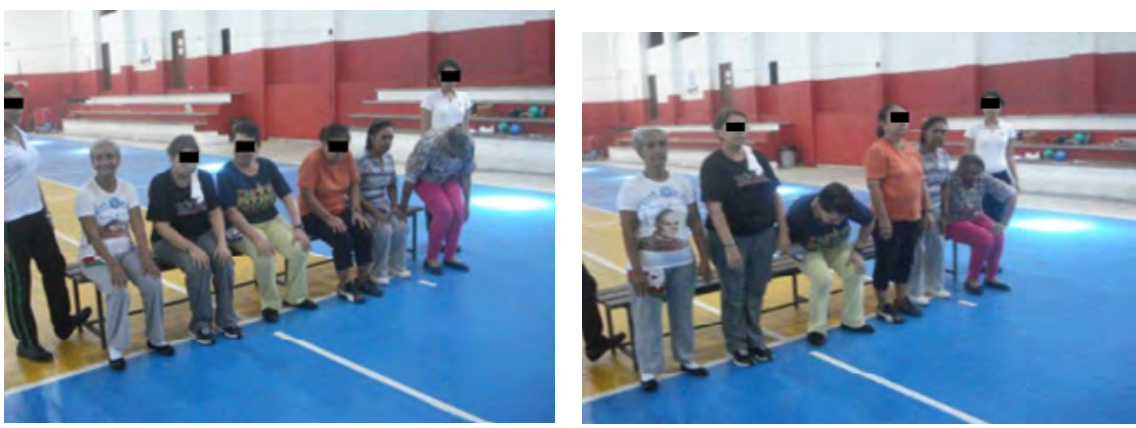


\section{Ejercicio No. 5.12}

Fortalecimiento de cuádriceps. Se fija un extremo de la liga a la silla o al otro pie. El extremo contrario de la liga se fija al dorso del pie contrario y se extiende la pierna. Hacer 10 repeticiones. Luego, con la otra pierna.

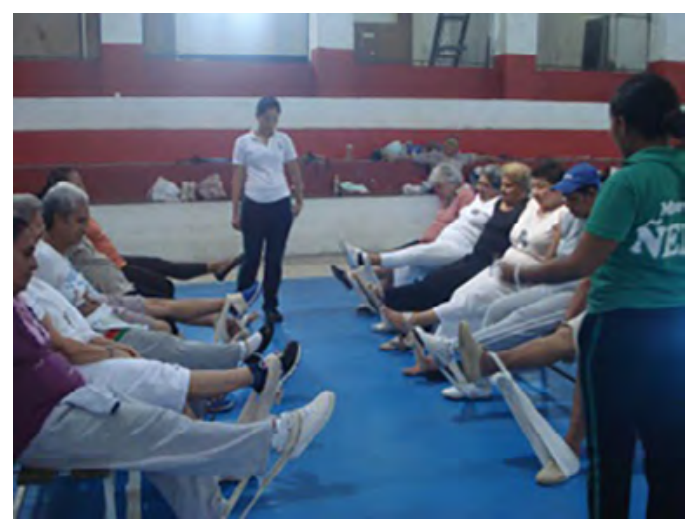




\section{Ejercicio No. 5.13}

Los ejercicios de sentarse y levantarse una o dos veces por día aumentan la fuerza de los tobillos, piernas y caderas, y ayudan al cuerpo a adaptarse a los cambios de posición sin marearse luego de haber estado sedentario durante largo tiempo.

Sentado en una silla (preferible sin descansabrazos) colocar las manos sobre las rodillas o cruzarlas en el pecho. Ponerse en posición de pie utilizando solo la fuerza de las extremidades inferiores sin apoyarse con las manos sobre las piernas u otro objeto. Hacer de 5 a 10 repeticiones.
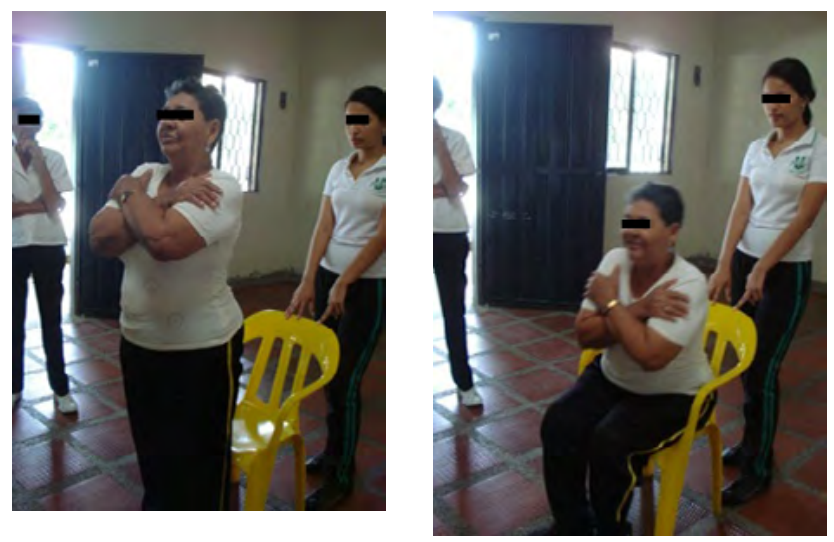


\section{Ejercicio No. 5.14}

Acostado de lado, descansando la cabeza sobre el brazo. Elevar la pierna superior, sin doblarla, lo más que sea posible. Hacer de 5 a 10 repeticiones. Acostarse sobre el lado contrario y repetir el ejercicio con la otra pierna.
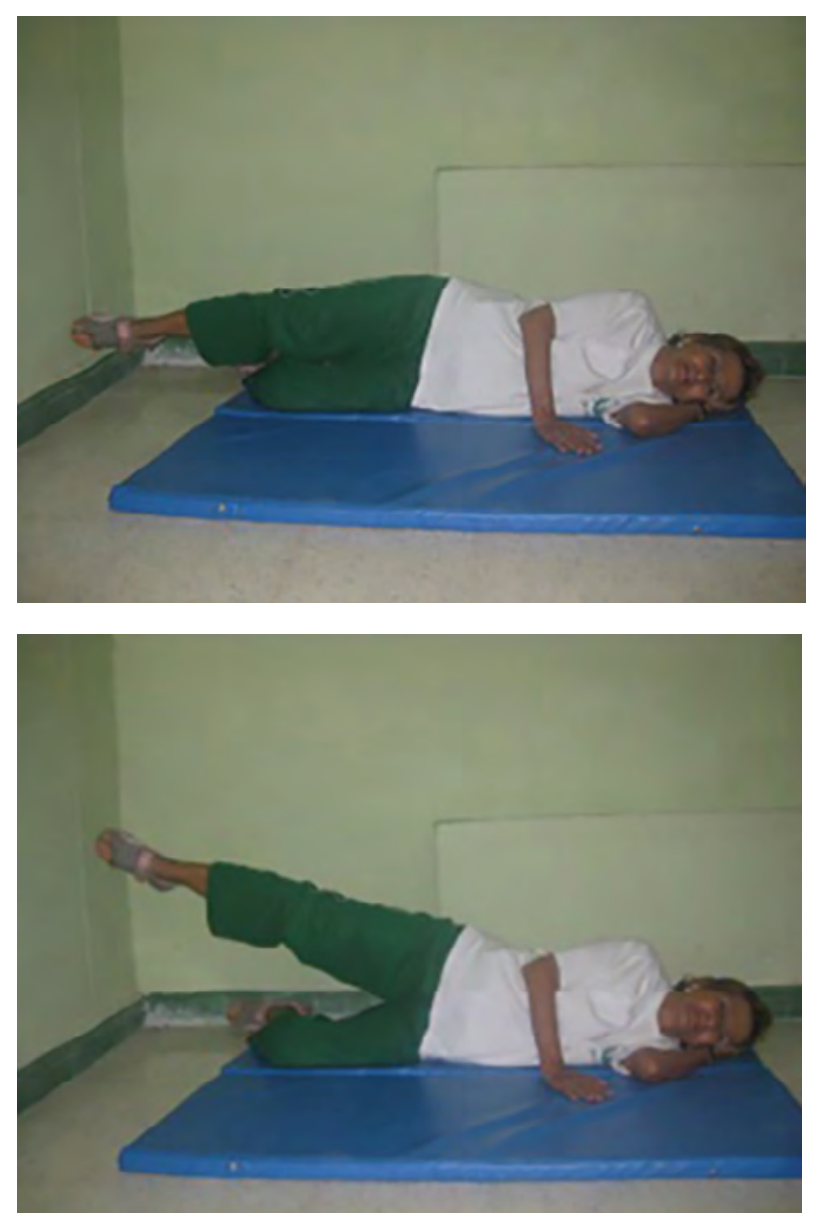


\section{Ejercicio No. 5.15}

Acostado boca abajo, apoyándose en las manos y los brazos, con las piernas estiradas, elevar la pierna derecha, sin flexionar la rodilla tanto como sea posible hasta sentir la tensión en los músculos posteriores.

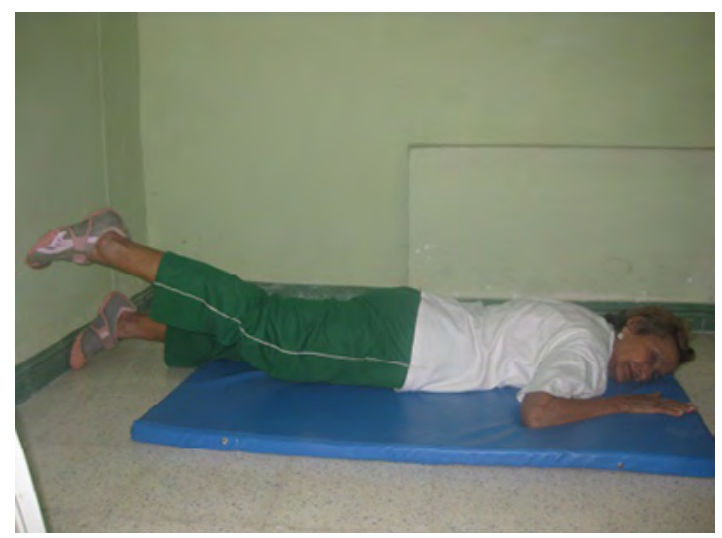




\section{VUELTA A LA CALMA}

Es llevar los signos vitales al estado inicial previo al ejercicio, lo cual se puede lograr por ejemplo a través de una caminata suave y movimientos de grandes rangos de los brazos.

\section{Secuencia del programa de ejercicios para 12 sesiones (1 mes)}

\begin{tabular}{|c|c|c|c|c|c|c|}
\hline \multirow[b]{2}{*}{ '. } & \multirow{2}{*}{ 总 } & \multicolumn{4}{|c|}{ PARTE CENTRAL } & \multirow[b]{2}{*}{ 牙 } \\
\hline & & $\begin{array}{c}\text { Estiramiento } \\
\text { Cuello: C } \\
\text { Tronco: T } \\
\text { Miembros sup: } \\
\text { MMSS } \\
\text { Miembros inf: MMII } \\
\end{array}$ & $\begin{array}{l}\text { Ejercicios } \\
\text { Coordi- } \\
\text { nacióny } \\
\text { equilibrio }\end{array}$ & $\begin{array}{c}\text { Parte } \\
\text { aeróbica }\end{array}$ & $\begin{array}{l}\text { Ejercicio } \\
\text { fuerza }\end{array}$ & \\
\hline 1 & $\begin{array}{l}10 \\
\text { min. }\end{array}$ & $\begin{array}{c}10 \mathrm{~min} . \\
\text { C: } 1.1 \\
\text { T: } 1.6 \\
\text { MMSS: } 1.2 \\
\text { MMII: } 1.7\end{array}$ & $\begin{array}{c}10 \mathrm{~min} . \\
2.1\end{array}$ & $\begin{array}{c}15 \mathrm{~min} . \\
4.1\end{array}$ & $\begin{array}{c}10 \mathrm{~min} \\
5.1\end{array}$ & $\begin{array}{l}10 \text { min. } \\
\text { Caminata suave, } \\
\text { movimientos } \\
\text { circulares de } \\
\text { MMSS }\end{array}$ \\
\hline 2 & $\begin{array}{l}10 \\
\text { min. }\end{array}$ & $\begin{array}{c}10 \mathrm{~min} . \\
\text { C: } 1.1 \\
\text { T: } 1.6 \\
\text { MMSS: } 1.3 \\
\text { MMII: } 1.8\end{array}$ & $\begin{array}{c}10 \mathrm{~min} . \\
2.2\end{array}$ & $\begin{array}{c}15 \min . \\
4.2\end{array}$ & $\begin{array}{c}10 \min \\
5.1 \\
5.2\end{array}$ & $\begin{array}{l}10 \text { min. } \\
\text { Caminata suave, } \\
\text { movimientos } \\
\text { circulares de } \\
\text { MMSS }\end{array}$ \\
\hline 3 & $\begin{array}{l}10 \\
\text { min. }\end{array}$ & $\begin{array}{c}10 \mathrm{~min} . \\
\text { C: } 1.1 \\
\text { T: } 1.6 \\
\text { MMSS: } 1.4 \\
\text { MMII: } 1.9\end{array}$ & $\begin{array}{c}10 \mathrm{~min} . \\
2.3 \\
3.1\end{array}$ & $\begin{array}{c}15 \mathrm{~min} . \\
4.3\end{array}$ & $\begin{array}{c}10 \mathrm{~min} \\
5.9 \\
5.10\end{array}$ & $\begin{array}{l}10 \text { min. } \\
\text { Caminata suave, } \\
\text { movimientos } \\
\text { circulares de } \\
\text { MMSS }\end{array}$ \\
\hline 4 & $\begin{array}{l}10 \\
\min .\end{array}$ & $\begin{array}{c}10 \mathrm{~min} . \\
\text { C: } 1.1 \\
\text { T: } 1.6 \\
\text { MMSS: } 1.5 \\
\text { MMII: } 1.10\end{array}$ & $\begin{array}{c}10 \mathrm{~min} . \\
2.4 \\
3.2\end{array}$ & $\underset{4.1}{15 \mathrm{~min} .}$ & $\begin{array}{c}10 \mathrm{~min} \\
5.11 \\
5.12\end{array}$ & $\begin{array}{l}10 \text { min. } \\
\text { Caminata suave, } \\
\text { movimientos } \\
\text { circulares de } \\
\text { MMSS }\end{array}$ \\
\hline 5 & $\begin{array}{c}10 \\
\text { min. }\end{array}$ & $\begin{array}{c}10 \mathrm{~min} . \\
\text { C: } 1.1 \\
\text { T: } 1.6 \\
\text { MMSS: } 1.2 \\
\text { MMII: } 1.11\end{array}$ & $\begin{array}{c}10 \mathrm{~min} . \\
2.5 \\
3.3\end{array}$ & $\begin{array}{c}15 \min . \\
4.2\end{array}$ & $\begin{array}{c}10 \min \\
5.3 \\
5.4\end{array}$ & $\begin{array}{l}10 \text { min. } \\
\text { Caminata suave, } \\
\text { movimientos } \\
\text { circulares de } \\
\text { MMSS }\end{array}$ \\
\hline
\end{tabular}




\begin{tabular}{|c|c|c|c|c|c|c|}
\hline \multirow[b]{2}{*}{ 긍 } & \multirow{2}{*}{ 兽 } & \multicolumn{4}{|c|}{ PARTE CENTRAL } & \multirow[b]{2}{*}{ 氞㜽 } \\
\hline & & $\begin{array}{c}\text { Estiramiento } \\
\text { Cuello: } \mathrm{C} \\
\text { Tronco: T } \\
\text { Miembros sup: } \\
\text { MMSS } \\
\text { Miembros inf: MMII }\end{array}$ & $\begin{array}{l}\text { Ejercicios } \\
\text { Coordi- } \\
\text { nacióny } \\
\text { equilibrio }\end{array}$ & $\begin{array}{c}\text { Parte } \\
\text { aeróbica }\end{array}$ & $\begin{array}{c}\text { Ejercicio } \\
\text { fuerza }\end{array}$ & \\
\hline 6 & $\begin{array}{l}10 \\
\text { min. }\end{array}$ & $\begin{array}{c}10 \text { min. } \\
\text { C: } 1.1 \\
\text { T: } 1.6 \\
\text { MMSS: } 1.3 \\
\text { MMII: } 1.12\end{array}$ & $\begin{array}{c}10 \min . \\
2.6 \\
3.4\end{array}$ & $\begin{array}{c}15 \min . \\
4.3\end{array}$ & $\begin{array}{c}10 \mathrm{~min} \\
5.9 \\
5.10\end{array}$ & $\begin{array}{l}10 \text { min. } \\
\text { Caminata suave, } \\
\text { movimientos } \\
\text { circulares de } \\
\text { MMSS }\end{array}$ \\
\hline 7 & $15 \mathrm{~min}$. & $\begin{array}{c}10 \text { min. } \\
\text { C: } 1.1 \\
\text { T: } 1.6 \\
\text { MMSS: } 1.4 \\
\text { MMIII: } 1.13\end{array}$ & $\begin{array}{c}10 \min . \\
2.7 \\
3.5\end{array}$ & $\begin{array}{c}15 \mathrm{~min} . \\
4.1\end{array}$ & $\begin{array}{c}10 \min . \\
5.13 \\
5.14\end{array}$ & $\begin{array}{l}10 \text { min. } \\
\text { Caminata suave, } \\
\text { movimientos } \\
\text { circulares de } \\
\text { MMSS }\end{array}$ \\
\hline 8 & $15 \mathrm{~min}$. & $\begin{array}{l}10 \mathrm{~min} . \\
\text { C: } 1.1 \\
\text { T: } 1.6 \\
\text { MMSS: } 1.5 \\
\text { MMII: } 1.7\end{array}$ & $\begin{array}{c}10 \mathrm{~min} . \\
2.8 \\
3.8-3.9\end{array}$ & $\underset{4.2}{15 \mathrm{~min}}$ & $\begin{array}{c}10 \min . \\
5.5 \\
5.6\end{array}$ & $\begin{array}{l}10 \text { min. } \\
\text { Caminata suave, } \\
\text { movimientos } \\
\text { circulares de } \\
\text { MMSS }\end{array}$ \\
\hline 9 & $15 \mathrm{~min}$. & $\begin{array}{c}10 \text { min. } \\
\text { C: } 1.1 \\
\text { T: } 1.6 \\
\text { MMSS: } 1.2 \\
\text { MMII: } 1.8\end{array}$ & $\begin{array}{c}10 \min . \\
2.9 \\
3.10-3.11\end{array}$ & $\begin{array}{c}15 \min . \\
4.3\end{array}$ & $\begin{array}{l}5.15 \\
5.16\end{array}$ & $\begin{array}{l}10 \text { min. } \\
\text { Caminata suave, } \\
\text { movimientos } \\
\text { circulares de } \\
\text { MMSS }\end{array}$ \\
\hline 10 & $15 \mathrm{~min}$ & $\begin{array}{c}10 \text { min. } \\
\text { C: } 1.1 \\
\text { T: } 1.6 \\
\text { MMSS: } 1.3 \\
\text { MMII: } 1.9\end{array}$ & $\begin{array}{c}10 \mathrm{~min} . \\
2.10 \\
3.12-3.13\end{array}$ & $\begin{array}{c}15 \mathrm{~min} . \\
4.1\end{array}$ & $\begin{array}{l}5.7 \\
5.8\end{array}$ & $\begin{array}{l}10 \text { min. } \\
\text { Caminata suave, } \\
\text { movimientos } \\
\text { circulares de } \\
\text { MMSS }\end{array}$ \\
\hline 11 & $15 \mathrm{~min}$ & $\begin{array}{l}10 \mathrm{~min} . \\
\text { C: } 1.1 \\
\text { T: } 1.6 \\
\text { MMSS: } 1.4 \\
\text { MMII: } 1.10\end{array}$ & $\begin{array}{c}10 \text { min. } \\
2.11 \\
3.14-3.15\end{array}$ & $\begin{array}{c}15 \mathrm{~min} . \\
4.2\end{array}$ & $\begin{array}{l}5.1 \\
5.2\end{array}$ & $\begin{array}{l}10 \text { min. } \\
\text { Caminata suave, } \\
\text { movimientos } \\
\text { circulares de } \\
\text { MMSS }\end{array}$ \\
\hline 12 & $15 \mathrm{~min}$ & $\begin{array}{l}10 \mathrm{~min} . \\
\text { C: } 1.1 \\
\text { T: } 1.6 \\
\text { MMSS: } 1.5 \\
\text { MMII: } 1.11\end{array}$ & $\begin{array}{c}10 \mathrm{~min} . \\
2.12 \\
3.16-3.17\end{array}$ & $\underset{4.3}{15 \mathrm{~min}}$ & $\begin{array}{l}5.9 \\
5.10\end{array}$ & $\begin{array}{l}10 \text { min. } \\
\text { Caminata suave, } \\
\text { movimientos } \\
\text { circulares de } \\
\text { MMSS }\end{array}$ \\
\hline
\end{tabular}




\section{REFERENCIAS BIBLIOGRÁFICAS}

1. ACSM. ACSM's Guidelines for exercise testing and prescription. Baltimore, Philadelphia: LWW; 2009.

2. Arias-Vázquez $P$, Balam-De la Vega V, Sulub-Herrera A, Carrillo-Rubio J, Ramírez-Meléndez A. Beneficios clínicos y prescripción del ejercicio en la prevención cardiovascular primaria: Revisión. Rev Mex Med Fis Rehab 2013;25(2):63-72.

3. Castañer M, Saüch GF, Camerino O, Sánchez-Algarra $P$, Anguera M. Percepción de la intensidad al esfuerzo: Un estudio multi-method en actividad física. Cuadernos de Psicología del Deporte. 2014;15(1); 83-88.

4. Chávez Samperio J, Lozano Dávila M, Lara Esqueda A, Velázquez Monroy O. La Actividad Física Y El Deporte En El Adulto Mayor. Bases fisiológicas. Disponible en http://www.salud.gob.mx/ unidades/cdi/documentos/DOCSAL7516.pdf

5. Peña GG. Si las escalas de percepción de esfuerzo son fiables ¿por qué no las utilizamos?. 2013 Disponible en: https://g-se. $\mathrm{com} / \mathrm{si}$-las-escalas-de-percepcion-de-esfuerzo-son-fiables-por-que-no-las-utilizamos-bp-q57cfb26d3588c

\section{Cómo citar este artículo}

Quintero Cruz MV, Pinillos Patiño Y. Diseño de un programa de ejercicio para el adulto mayor. In Quintero Cruz MV, Pinillos Patiño Y, Herazo Beltrán AY, Vidarte Claros JA, Cardeño Sanmiguel GM, Morales Castro YR. Ejercicio físico para la condición física funcional en el adulto mayor: Estrategia de intervención. Barranquilla: Universidad Simón Bolívar; 2017. p. $83-130$. 